\title{
Changes in Autonomic Nervous System Activity and Mood of Healthy People after Mindfulness Art Therapy Short Version
}

\author{
Michiyo Ando ${ }^{*}$, Sayoko Ito ${ }^{2}$ \\ ${ }^{1}$ St. Mary's College, Kurume City, Japan \\ ${ }^{2}$ Kyoto Seizan Junior College, Nagaoka City, Japan \\ Email: *andou@st-mary.ac.jp
}

Received 6 October 2015; accepted 22 February 2016; published 25 February 2016

Copyright (C) 2016 by authors and Scientific Research Publishing Inc.

This work is licensed under the Creative Commons Attribution International License (CC BY).

http://creativecommons.org/licenses/by/4.0/

(c) (i) Open Access

\begin{abstract}
The aim of this study was to investigate changes in autonomic nervous system (ANS) activity and mood caused by Mindfulness Art Therapy Short version (MATS). The participants were 20 Japanese college students who were separated into high and low risk groups based on the median score of the General Health Questionnaire (GHQ). MATS consisted of mindfulness exercise and making of art in one session. ANS activity (TP: total energy, LF/HF: sympathetic nervous, HF: parasympathetic nervous system, LF: both sympathetic and parasympathetic) and mood (TA: tension arousal, EA: energy arousal) were measured psychologically before and after MATS. In the high risk group, TP significantly decreased and LF, HF, and LF/HF did not change significantly; while TA significantly decreased and EA significantly increased. In the low risk group, TP and LF significantly increased and HF and LF/HF did not change significantly; while TA significantly decreased and EA showed a non-significant increase. These results suggest that MATS affects the ANS differently for participants with different states of mental health, and particularly promotes activity in low-risk participants. Psychologically, MATS decreased tension or anxiety and increased energy. These findings justify further use of this therapy.
\end{abstract}

\section{Keywords}

Mindfulness, Art Therapy, Mood, Autonomic Nervous System

\section{Introduction}

Cancer patients experience physical, social, psychological, and spiritual pain. Art therapy is a psychotherapy

${ }^{*}$ Corresponding author. 
that has been shown to be effective for anxiety [1], depression [2], spiritual well-being [3], and somatic symptoms [4] in cancer patients. Mindfulness is another effective approach, and Kabat-Zin [5] developed the Mindfulness-Based stress Reduction (MBSR) program based on the principle of mindfulness, which is defined as moment-to-moment, present-centered, purposive non-judgmental awareness. MBSR is effective for improvement of quality of life and mood [6] and for post-traumatic growth and spirituality [7].

Mindfulness-Based Art Therapy (MBAT) integrates mindfulness and art therapy, and leads to significant decreases in symptoms of distress and increased quality of life [8]. Physiologically, MBAT for 8 weeks increases cerebral blood flow (CBF) and there is a correlation between increased CBF and decreased anxiety [9]. However, the duration of MBAT of 8 weeks in a group format may to be hard for patients with advanced cancer, and a simpler version, the Mindfulness Art Therapy Short version (MATS), was developed [10]. MATS is effective for alleviation of tension-anxiety, depression and fatigue, and elevation of vigor in healthy people [11].

Previous studies of MATS have used questionnaires as psychological indicators, and thus the physiological changes induced by MATS remain unclear. Autonomic nervous system (ANS) activity is a useful physiological indicator, since this reflects heart rate variability (HRV), which in turn reflects the subtle relationship between the heart and lung. In addition, brain scans and EEG studies require immobility [12], but this is not possible for an action-based form of mindfulness or therapeutic use of art; however, resting HRV has become an accepted marker for vagal tone and neurobiological correlates of internal composure. It is also likely that the state of mental health of participants affects MATS because participants impressions were divided into two areas such that some experiences were much more focused on the mind in a previous study [11]. Thus, we investigated changes in the ANS and mood in participants based on their state of mental health.

To measure mood, we chose a scale which measure present states. And to measure their mental health, we chose a scale which is used in worldwide studies.

\section{Methods}

\subsection{Participants}

The participants were 20 college students at a single college in Japan. They were recruited by advertisement of the study and their participation was voluntary. The inclusion criterion was age $>20$ years old and the exclusion criterion was the presence of severe mental problems. The study was approved by the college ethical board.

\subsection{Measures}

The General Health Questionnaire 30 (GHQ30) was used to measure mental health state, including physical state [13] [14]. Autonomic nervous system (ANS) activity was evaluated using TAS 9 (YKC Corp.), which uses heart rate to measure total energy (TP) of the sympathetic and parasympathetic nervous system level (LF: low frequency), parasympathetic level (HF: high frequency), and the balance of sympathetic and parasympathetic nerve levels and the sympathetic nervous system (LF/HF). Mood was measured using the Japanese UWIST Mood Adjective Checklist (JUMACLE) [15]. This consists of 10 items for tension arousal (TA), which includes tension or anxiety; and 10 items for energy arousal (EA), which includes activity or vigor. Each item is scored by each participant from 1 to 4 on a Likert scale. A high score indicates high TA or EA.

The procedure for the Mindfulness Art Therapy-Short version program has been described elsewhere [11]. The intervention includes mindfulness and art therapy of 90 minutes in one session. Participants are instructed on mindfulness with assistance from a CD developed by a Yoga specialist and a clinical psychologist. First, participants exercised mindfulness by listening to the CD while receiving support from the clinical psychologist. This required about 20 minutes. The simple instructions in the $\mathrm{CD}$ were designed to make the participants aware of mind and body without judgment. After mindfulness practice, participants were given art materials including clay, collage (fancy paper, felt glue sticks, magazines), drawing instruments (colored pencils, pastel chalks, pencils, water colors), and sketch books and invited to make art by expressing their feelings or emotions freely.

\subsection{Procedures}

Students were recruited by an advertisement in the college. If a student was interested in the research, he or she telephoned the researchers and made a reservation. Each participant received MATS individually with a clinical psychologist. First, the clinical psychologist explained the study in more detail. If the participant continued to 
agree to participation, they gave signed informed consent and the study was started. Each participant completed the GHQ and JUMACLE questionnaires, and ANS activity was measured by TAS9 via a sensor. After MATS, the JUMACLE was completed again and ANS activity was measured for a second time.

\subsection{Analysis}

Statistical analysis was conducted with SPSS ver. 21.0 (Japanese version) for Windows (SPSS Inc.). Scores for ANS activity and JUMACLE pre- and post-intervention were compared by t-test. All reported p values are 2-tailed and $\mathrm{p}<0.05$ was taken to indicate a significant level in all analyses.

\subsection{Abbreviations}

In this paper, we use words as followings:

1) Mindfulness Art Therapy Short version: MATS

2) Autonomic Nervous System: ANS

3) Heart Rate Variability: HRV

4) Total energy: TP

5) Low Frequency: LF

6) High Frequency: HF

7) Balance of sympathetic and parasympathetic nerve levels: LF/HF

8) Tension Arousal: TA

9) Energy arousal: EA

\section{Results}

The demographic data are shown in Table 1, and the results for HRV and mood are shown in Table 2 to Table 5. In the high risk group, there were no significant differences between pre- and post-MATS values for LF, HF, and LF/HF. The score for TP significantly decreased from 7.28 to $7.01(\mathrm{p}<0.05)$. TA significantly decreased from 18.8 to $13.4(\mathrm{p}<0.005)$ and EA significantly increased from 30 to $34(\mathrm{p}<0.05)$. In the low risk group, LF significantly increased from 5.08 to $5.92(\mathrm{p}<0.009)$ and TP significantly increased from 6.73 to $7.12(\mathrm{p}<$ 0.004). There were no significant differences in HF and LF/HF pre- and post-MATS. TA significantly decreased from 16.5 to12.2 $(\mathrm{p}<0.001)$ and there was no significant difference in EA pre- and post-MATS.

\begin{tabular}{|c|c|c|}
\hline Item & Number & Percentage \\
\hline \multicolumn{3}{|l|}{ Gender: } \\
\hline Male & 4 & 20 \\
\hline Female & 16 & 80 \\
\hline \multicolumn{3}{|l|}{ Age: years } \\
\hline from 20 to 29 & 12 & 60 \\
\hline from 30 to 39 & 4 & 20 \\
\hline from 40 to 49 & 4 & 20 \\
\hline \multicolumn{3}{|l|}{ Job: } \\
\hline No & 11 & 55 \\
\hline Yes & 9 & 45 \\
\hline (Student with Job) & & \\
\hline
\end{tabular}


Table 2. Pre- and post-intervention scores for ANS activities in the high risk group $(\mathrm{n}=10)$.

\begin{tabular}{ccccc}
\hline & Pre & Post & t-value & p value \\
\hline LF & $5.91 \pm 1.2$ & $5.43 \pm 0.7$ & 1.17 & $\mathrm{p}=0.27$ \\
HF & $6.07 \pm 1.0$ & $5.79 \pm 0.8$ & 1.13 & $\mathrm{p}=0.29$ \\
LF/HF & $0.98 \pm 0.2$ & $0.96 \pm 0.2$ & 0.23 & $\mathrm{p}=0.83$ \\
TP & $7.28 \pm 0.6$ & $7.01 \pm 0.5$ & 2.53 & $\mathrm{p}=0.03^{*}$ \\
\hline${ }^{*}<0.05$. & & &
\end{tabular}

Table 3. Pre- and post-intervention scores for subdomains of mood in the high risk group $(\mathrm{n}=10)$.

\begin{tabular}{ccccc}
\hline & Pre & Post & t-value & p value \\
TA & $18.8 \pm 4.7$ & $13.4 \pm 3.4$ & 3.70 & $\mathrm{p}=0.005^{* *}$ \\
EA & $30.0 \pm 5.1$ & $33.9 \pm 4.1$ & -2.5 & $\mathrm{p}=0.04^{*}$ \\
\hline
\end{tabular}

${ }^{*} \mathrm{p}<0.05,{ }^{* *} \mathrm{p}<0.01$

Table 4. Pre- and post-intervention scores for ANS activities in the low risk group $(n=10)$.

\begin{tabular}{ccccc} 
& Pre & Post & t-value & $\mathrm{p}$ value \\
\hline LF & $5.08 \pm 1.2$ & $5.92 \pm 1.2$ & -3.34 & $\mathrm{p}=0.009^{* *}$ \\
HF & $5.23 \pm 1.0$ & $5.55 \pm 1.0$ & -2.20 & $\mathrm{p}=0.056$ \\
LF/HF & $0.97 \pm 0.1$ & $1.09 \pm 0.2$ & -1.84 & $\mathrm{p}=0.10$ \\
TP & $6.73 \pm 0.7$ & $7.12 \pm 0.5$ & -3.83 & $\mathrm{p}=0.004^{* *}$ \\
\hline
\end{tabular}

${ }^{* *} \mathrm{p}<0.001$

Table 5. Pre- and post-intervention scores for subdomains of mood in the low risk group $(\mathrm{n}=10)$.

$\begin{array}{ccccc} & \text { Pre } & \text { Post } & \text { t-value } & \text { p value } \\ \text { TA } & 16.5 \pm 3.2 & 12.2 \pm 2.4 & 4.5 & \mathrm{p}=0.001^{* *} \\ \text { EA } & 32.6 \pm 4.2 & 33.8 \pm 4.4 & -0.7 & \mathrm{p}=0.49\end{array}$

${ }^{*} \mathrm{p}<0.05,{ }^{* *} \mathrm{p}<0.01$.

\section{Discussion}

The absence of significant difference in LF, HF, and LF/HF in the high risk group indicates that there were no significant physiological changes in the sympathetic and parasympathetic nervous systems in this group. However, the significant decrease in TP shows that total energy in the ANS decreased. This may be because performance of mindfulness and making art for 90 minutes consumed their physical strength. For the psychological indicators, the significant decrease in TA indicates a decrease in tension or anxiety, while the significant increase in EA indicates an increase in energy. Therefore, the high-risk participants were activated and revitalized by MATS. That is, physiologically, the participants in the mental high risk group might have been fatigued; however, psychologically, they were relaxed and activated after MATS. A previous study showed decreased sympathetic activity and increased parasympathetic activity after back massage in healthy people with high sympathetic activity at baseline [16]. In contrast, in the current study, there was no significant difference in HF and LF because MATS is an active intervention, whereas an intervention such as back massage is passive.

In the low risk group, the significant increase of LF suggests an increase in sympathetic activity. Moreover, the LF/HF of $>1.0$ suggests that sympathetic activity was activated more than parasympathetic activity. The significant increase in TP indicates that the total energy of the ANS also increased. That is, for the low-risk mental health group, MATS was a physiological activator. Compared with the high risk group, MATS may be more 
Table 6. Merit of the MATS for cancer patients.
1) A participant can conduct individually.
2) Duration of a session time is about from 60 minutes to 90 minutes, and participants can complete easily.
3) Mindfulness part in the MATS require little physical movement, and participants with weak physical strength may do.
4) A participant can conduct event though they are on the bed.
5) They can use CD for mindfulness, and they can conduct when a supporter is absent.

effective for elevating ANS activity in healthy people with low mental health risk. One of the reasons for this effect may be that this therapy increases cerebral blood flow [9], which then activates brain function and increases ANS activity.

Psychologically, the significant decrease in TA in the low risk group indicates a significant decrease intension or anxiety, as also found for the high risk group. Thus, MATS is useful in this respect for all participants. In contrast, energy was high at baseline and did not increase (no significant change in EA) in the low risk group. These changes show that participants in this group were activated and energetic, and had decreased tension after MATS. This is consistent with the finding by Monti et al. [17] that anxiety decreased after mindfulness art therapy in cancer patients.

Lastly, we estimate merits of the MATS for cancer patients. This method may be useful for cancer patients in these points like Table 6. Moreover, this study may be important before conducting the MATS for cancer patients to confirm potentiality measuring automatic nervous system. And from this study we estimate merits for cancer patients; 1) since the MATS alleviate tension-anxiety (TA) for healthy people, it may alleviate tensionanxiety for cancer patients, 2) it elevated energy of healthy people physiologically in the low risk group, it may elevate energy of cancer patients with low mental health risk, 3) since mental health like depression or anxiety related with spirituality in the previous study [18], it might affect spirituality of cancer patients. In future study, we need to confirm these points.

The study is limited by the small number of participants and the absence of a randomized controlled design. Therefore, evaluation of the robustness and reliability of the findings will require a further study in more subjects in a randomized controlled trial.

\section{Conclusions}

We investigated changes in the ANS and mood in participants by the MATS based on their state of mental health.

1) In the high risk group, TP significantly decreased and LF, HF, and LF/HF did not change significantly; while TA significantly decreased and EA significantly increased.

2) In the low risk group, TP and LF significantly increased and HF and LF/HF did not change significantly; while TA significantly decreased and EA showed a non-significant increase.

3) These results suggest that MATS affects the ANS differently for participants with different states of mental health, and particularly promotes activity in low-risk participants.

4) Psychologically, MATS decreased tension or anxiety and increased energy. These findings justify further use of this therapy.

\section{Acknowledgements}

This study was supported by JSPS KAKENHI grant number 245309010001 .

\section{References}

[1] Oster, I., Svensk A.C., Magnusson E., Thyme, K.E., Sjodin, M., Astrom, S. and Lindh, J. (2006) Art Therapy Improves Coping Resources: A Randomized, Controlled Study among Women with Breast Cancer. Palliative and Supportive Care, 4, 54-64. http://dx.doi.org/10.1017/S147895150606007X

[2] Bar-Sela, G., Atid, L., Danos, S., Gabay, N. and Epelbaum, R. (2007) Art Therapy Improved Depression and Influenced Fatigue Levels in Cancer Patients on Chemotherapy. Psychooncology, 16, 980-984. http://dx.doi.org/10.1002/pon.1175 
[3] Vella, E. and Budd, M. (2011) Pilot Study: Retreat Intervention Predicts Improved Quality of Life and Reduced Psychological Distress among Breast Cancer Patients. Complimentary Therapies in Clinical Practice, 17, 209-214. http://dx.doi.org/10.1016/j.ctcp.2011.01.005

[4] Thyme, K.E., Sundin, E.C. and Wiberg, B. (2009) Individual Brief Art Therapy Can Be Helpful for Women with Breast Cancer: A Randomized Controlled Clinical Study. Palliative and Supportive Care, 7, 87-95. http://dx.doi.org/10.1017/S147895150900011X

[5] Kabat-Zinn, J. (1990) Full Catastrophe Living: Using the Wisdom of Your Body and Mind to Face Stress, Pain and Illness. Delacourt, New York.

[6] Carlson, L.E., Speca, M., Patel, K.D. and Goodey, E. (2004) Mindfulness-Based Stress Reduction in Relation to Quality of Life, Mood, Symptoms of Stress and Levels of Cortisol, Dehydroepiandrosterone Sulfate (DHEAS) and Melatonin in Breast and Prostate Cancer Outpatients. Psychoneuroendocrinology, 29, 448-474. http://dx.doi.org/10.1016/S0306-4530(03)00054-4

[7] Garland, S.N., Carson, L.E., Cook, S., Lansdell, L. and Speca, M. (2007) A Non-Randomized Comparison of Mindfulness-Based Stress Reduction and Healing Arts Programs for Facilitating Post-Traumatic Growth and Spirituality in Cancer Outpatients. Supportive Care in Cancer, 15, 949-961. http://dx.doi.org/10.1007/s00520-007-0280-5

[8] Monti, D.A., Peterson, C. and Kunkel, S. (2006) A Randomized, Controlled Trial of Mindfulness-Based Art Therapy (MBAT) for Women with Cancer. Psycho-Oncology, 15, 363-373. http://dx.doi.org/10.1002/pon.988

[9] Monti, D.A., Kash, K.M., Kunkel, E.J., Brainard, G., Wintering, N., Moss, A.S., Rao, H., Zhu, S. and Newberg, A.B. (2012) Changes in Cerebral Blood Flow and Anxiety Associated with an 8-Week Mindfulness Program in Women with Breast Cancer. Stress \& Health, 28, 397-407. http://dx.doi.org/10.1002/smi.2470

[10] Ando, M., Kira, H. and Hayashida, Y. (2014) Potential Effect of Mindfulness Art Therapy Short Version on Mood in Patients with Advanced Cancer. Art Therapy, in press.

[11] Ando, M. and Ito, S. (2014) Potentiality of Mindfulness Art Therapy Short Version on Mood of healthy People. Health, 6, 1224-1229. http://dx.doi.org/10.4236/health.2014.611150

[12] Porges, S.W. (2011) The Polyvagal Theory: Neurophysiological Foundations of Emotions, Attachment, Communication, Self-Regulation. Norton, New York.

[13] Goldberg, D.P. and Hiller, V.E. (1979) A Scaled Version of the General Health Questionnaire. Psychological Medicine, 9, 139-145. http://dx.doi.org/10.1017/S0033291700021644

[14] Nakagawa Y. and Daibo, I. (1985) The General Health Questionnaires. Nihon Bunka Kagakusya, Tokyo.

[15] Sirasawa, S., Ishida, T., Hakoda, Y. and Haraguchi, M. (1999) The Effects of Energetic Arousal on Memory Search. The Japanese Journal of Psychonomic Science, 17, 93-99.

[16] Fujita, Y., Kinouchi, C., Abe, Y., Shimotake, Y. and Satou, N. (2013) Physiological and Psychological Effects of Back Massage on Japanese Adult Females. International Nursing Care Research, 12, 45-55.

[17] Monti, D.A., Kash, K.M., Kunkel, E.J., Moss, A., Mathews, M., Brainard, G., Anne, R., Leiby, B.E., Pequinot, E. and Newberg, A.B. (2013) Psychosocial Benefits of a Novel Mindfulness Intervention versus Standard Support in Distressed Women with Breast Cancer. Psychooncology, 22, 2565-2575. http://dx.doi.org/10.1002/pon.3320

[18] Ando, M., Morita, T., Akechi, T., Ito, S., Tanaka, M., Ifuku, Y. and Nakayam, K. (2009) The Efficacy of MindfulnessBased Meditation Therapy on Anxiety, Depression, and Spirituality in Japanese Patients with Cancer. Journal of Palliative Medicine, 40, 453-459. 